\title{
The Innovative Application of Chinese Element Printing Pattern in Fashion Design
}

\author{
Xiaoxiu Liu \\ Guangdong University of Science \& Technology, Dongguan, China, 523083
}

Keywords: Chinese elements; printing pattern; fashion design

\begin{abstract}
Fashion design is a complex and highly detailed process. In the traditional clothing design process, the choice of printing patterns is a very important part. The printing pattern can bring different energy and vitality to the whole piece of clothing, and can also present a lot of unique qualities. The process of patterning a dye or painting on a fabric is a fabric print. Printing is local dyeing and requires a certain degree of color fastness. In addition, printing is also one of the important ways of fabric decoration. This paper first introduces the history of the development of Chinese element printing patterns, then introduces the printing method of printing patterns, and finally the application of printing patterns in fashion design.
\end{abstract}

\section{Introduction}

As a traditional folk art form, the print motif is deeply rooted in ancient and long history and culture in China. With the development of the times, based on inheriting and absorbing the essence of its traditional art and technology, we explored new techniques and new artistic ideas, and opened up new directions for the application of printed patterns in modern apparel design ${ }^{[1]}$. The application of printed patterns in modern apparel design is divided into traditional applications and innovative applications. Traditional application refers to the application of a pattern of a print pattern itself to a garment design. Nowadays, print patterns can be interpreted in modern fashion to suit more modern aesthetic standards. The innovative application is to extract the most representative characteristic elements of the print pattern, break the reorganization, change the color, replace the pattern, try different techniques and other methods to combine it with the modern fashion design concept, as a separate design element. creation.

\section{History of the Development of Printing Patterns}

In China, as early as in the Neolithic Age, pottery printing was adopted. From the Zhou dynasty onwards, it was used for seals and mud, and even in the Spring and Autumn and the Warring States period, the relief printing was applied to fabrics, and it was quite high in the Western Han Dynasty. Unearthed printed color yarn is a combination of three pieces of relief printing and then painting. During the Sui and Tang Dynasties, a large number of printed fabrics had been transmitted to the Western Regions through the "Silk Road" and to the Japanese in the fifth and sixth centuries. At the same time as the development of letterpress printing, or at the same time, another type of printing method, sculpting and stencil printing, has emerged one after another, along with the letterpress printing.

This kind of printing technology, according to historical records, has already been known as the Qin and Han dynasties, which was then called "clips." During the Northern Wei Dynasty of the Northern and Southern Dynasties, this kind of process had a considerable scale. During the Sui and Tang dynasties, the technology became more sophisticated, and it had been able to produce advanced products such as the "Five-colored Jerusalem Dress" and invented the addition of screens on the stenciled version to solve the difficulties of printing closed circle patterns. In the Song Dynasty, the production of clip printing was specialized, and printed fabrics were very popular. The folder was introduced to Japan during the Sui and Tang dynasties; after the Song Dynasty, along with the development of maritime transportation, it was gradually taken to Western European countries ${ }^{[2]}$. On 
the eve of liberation, roller printing and "slurry printing" are used. After the liberation, the printing technology and production have been greatly developed. Mechanized productions such as lithographic screen printing and rotary screen printing have been developed. The printing process has been continuously improved. There are special printing, imitation printing, metal foil printing, and coating cover printing. Flicker printing, etc., have greatly improved the appearance and inherent quality of the fabric. Now printing has become a fashion element. Figures 1 to 3 below are some of the traditional Chinese print patterns.

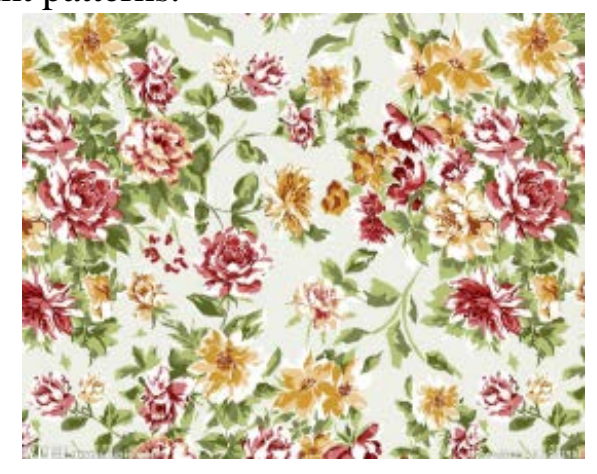

Figure 1

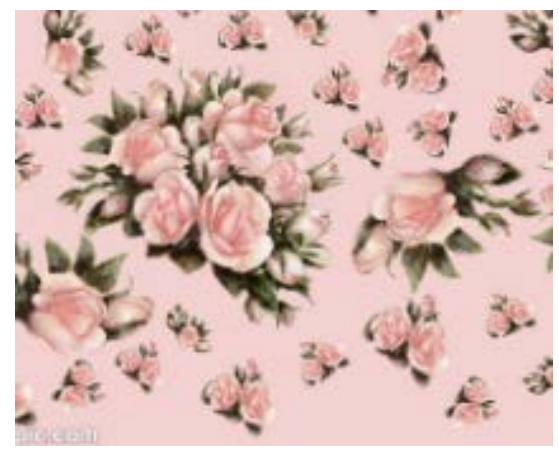

Figure 3

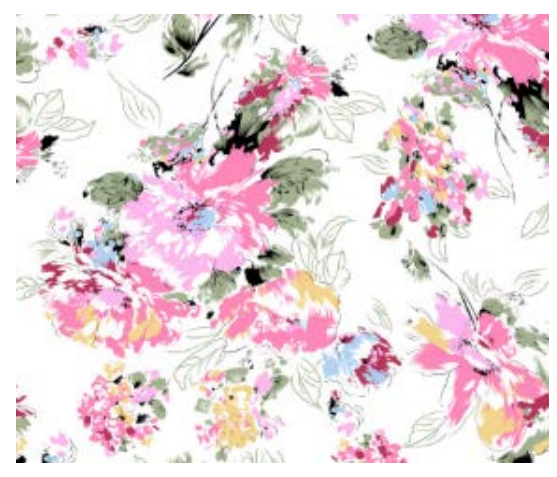

Figure 2

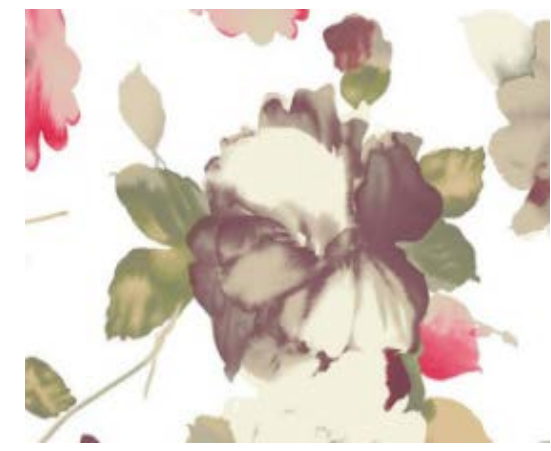

Figure 4

\section{Printing Methods of Printing Pattern}

\subsection{Direct printing}

One of the printing methods. The method of directly printing a flower pattern of various colors on a fabric is a direct printing. This printing process is the simplest and most common one of several printing methods. In the printing process, color pastes of various colors do not cause obstruction and damage. About $80 \%-90 \%$ of printed fabrics use this method. The law can print white flowers and Montreal flowers. According to the different requirements of the pattern, it is divided into three kinds: white land, full land and colored ground: the area of white land printing pattern is small, and the area of white land is large; the area of landscaping printing pattern is large, most of the fabric area is printed with patterns; the direct printing of ground areas Means to first dye a good color, and then printed on the pattern, commonly known as "cover printing." However, due to the color of the reason, generally use the same kind of color, similar to the color or shallow deep flowers are mostly, or the color at the overlapping color fading.

\subsection{Folding and discharging}

On fabrics that have been dyed, a paste containing a reducing agent or oxidizer is printed to destroy its color and partially reveal white or colored patterns. Color-dyed fabrics are printed with color paddles containing chemicals that can destroy color, and such chemicals are called discharge agents. Dyed propellers can also be added with chemically resistant dyes. This kind of discharge printing can get two kinds of effects, namely pulling white and pulling. 


\subsection{Folding protection}

On the fabric is printed with a paste to prevent the dyeing or coloration of the ground color dyes, and then dyeing to obtain a printing process of the colored floor cloth. The substance that prevents the dyeing in the printing paste is called a resist. The printing of a white pattern printed with a printing paste containing a dye-resistant agent is called anti-white printing; adding a dye or a pigment that is not affected by a dye-resistant printing agent into a color pattern is called a color printing prevention printing ${ }^{[3]}$. Firstly, the fabric is printed with a dye-resistive agent that can prevent the dye from being dyed, and then the color of the pad is dyed. The pattern is printed to prevent the color from being stained. The method is to prevent the dyeing and printing. This method can obtain three effects, namely White, color and part of the anti-dyed.

\section{Application of Printing in Fashion Design}

\subsection{Print pattern brings color innovation to fashion design.}

Color is one of the main factors in the recognition of objects, and it is also the most easily recognized feature of objects. Most of the folk crafts in our country use red, yellow, and green, three or more intense colors of high purity, pay attention to "hit color," "color-lined," and "evenness," and present a warm and jubilant festive atmosphere. People's wishes for a better life. The print pattern has always maintained its own unique features in a wide variety of handicrafts. The print patterns can be formed in various styles and styles and widely spread. Whether it is a traditional print with a historical atmosphere or a fashion pattern that incorporates modern trends, printing has become a symbol of China's fashion design. In the past millennia, printed patterns have been integrated into people's daily lives. Even the change of the dynasty will not change people's unique emotions on the print. Varied shapes are always colors that people cannot replace. However, with the development of the times, western dress culture has strongly impacted people's aesthetic tastes. Under the influence of the “westerly winds moving eastward”, people’s pattern ideas have changed. The new style of clothing is accompanied by more and more people's acceptance of the rich colors, printing patterns have gradually been forgotten in the corner. The protection and transmission of prints to adapt this traditional art to the development of the new era and satisfy people's new aesthetic needs are imperative for people to urgently solve. In the international market, the concept of pattern marketing has guided the development direction of the printing pattern innovation ${ }^{[4]}$. "Enterprises shall fully apply pattern representation techniques according to market characteristics, reflect the external characteristics of their products to conduct marketing mix, and meet the specific needs of customers." The American marketing industry has summed up the seven-second rule: Consumers will decide their willingness to buy within seven seconds, and patterns account for a large proportion. Therefore, if the pattern selling point as a marketing tool will bring huge market benefits. In the current consumer market, people are driven by the need for color diversity. Pattern innovation has become one of the means of inheritance and innovation of printed patterns.

\subsection{Print pattern brings pattern innovation to fashion design.}

Traditional prints emphasize integrity, decorativeness, and implication, and the arrangement uses more standardized forms of symmetry, such as the "happy meet" formed by the concept of Tai Chi, the form of the Jiugong and Mi Zi formed by the concept of Five Elements, and the composition of the whole pattern. Seek whole and seek full. Modern designs often exhibit asymmetric, changeable, and contradictory spatial effects. To make innovations in the pattern of prints, it is necessary to start from breaking the copy of the traditional patterns and to get rid of the fixed patterns, using the modern concepts and forms of expression to innovate the designs of traditional print patterns, and to meet the modernization through decomposition-refining-recombination. The aesthetic standards. The most important feature of the pattern of printing patterns that distinguishes it from other traditional patterns is that the arrangement of points and lines constitutes a pattern. Various types of point patterns such as circles, sectors, triangles, squares, line segments, irregular shapes, etc. are all freely used. Make the point of expression and pattern to achieve a unified realm. The composition of the dotted line is the 
most recognizable characteristic of the printed pattern, and therefore the expression of the dotted line cannot be ignored in considering the innovative design on the printed pattern.

\subsection{Print pattern brings connotation innovation to fashion design.}

The symbol of auspicious designs and the theme of narrative are the main manifestations of printed designs. In modern dress design, the symbolism of the pattern is weakening. If the modern pop pattern is grafted into the traditional printing pattern innovation, the traditional printing pattern's craft features are retained, making it more in line with the popular aesthetic requirements. Geometric patterns are typical elements in modern design, while Op Art style visual and visual errors are representatives of modern art styles. They are frequently used by major fashion designers in recent years, and there are also lines in Op Art patterns ${ }^{[5]}$. The composition. It is similar to the geometric patterns of traditional printing patterns. Its pattern composition is more conducive to the performance of traditional printing patterns engraving and printing.

\section{Conclusion}

The printing pattern is a design element with the characteristics of the traditional culture of the nation. The use of modern design needs to continuously inject new innovative elements that are in line with the progress of the times. Mr. Zhang Daoyi once said: "No matter how good the ancient patterns are; it cannot substitute for today's creation. The use of cultural heritage in the real sense is a reference to new creations. It is to use the mirrors of the predecessors to see their own ways and make their own experiences. Rich." Modern Chinese fashion designs need traditional folk art such as prints to moisturize. At the same time, printed designs also need to be developed in the new era with innovative designs and have a place in modern fashion.

\section{References}

[1] Pan Chunyu; Gao Weidong. Application of Sporadic Colors in Innovative Design of Blue Calico[A]; [C];2009

[2] Wu Yuanxin. The status quo of traditional blue print cloth [N]; China Culture News; 2011

[3] Tang Fan. Study on the pattern and art form of blue calico in Xiangxi [D]; Hunan University of Technology;2009

[4] Wang Yisai. Application of Chinese Traditional Blue Calico in Fashion Design[D]; Zhejiang Agriculture and Forestry University;2011

[5] Zhang Yang. Analysis of the Application of Folk Dress Pattern in Silk Print Design[J]; Artwork Kam; 2016-02 